\title{
Calidad de vida y su correlación con los precios del suelo: aproximación a la segregación residencial en Bogotá*
}

\begin{tabular}{|l|l|l|}
\hline Fecha de recepción: 17 de febrero $2016 \quad$ Fecha de aceptación: 7 de octubre 2016 & Disponible en línea: 12 de mayo de 2017 \\
\hline José Mario Mayorga & \\
\hline $\begin{array}{l}\text { Magíster en Planeación } \\
\text { Urbana y Regional }\end{array}$ & Catedrático de la Pontificia Universidad Javeriana & jmmayorgahenao@gmail.com \\
\hline
\end{tabular}

\section{Diva Marcela García}

Doctoranda en Demografía $\quad$ Catedrática de la Pontificia Universidad Javeriana $\quad$ mgarcia@ced.uab.es

\section{Laura Hernández}

Maestranda en Geografía

Resumen Con el artículo se pretende caracterizar, mediante datos empíricos, la concentración de condiciones de alta y baja calidad de vida en la ciudad de Bogotá en 2005, así como la georreferenciación de los precios del suelo, en una escala de alta proximidad geográfica. Lo anterior, a la luz del concepto de segregación residencial, en busca de identificar sus formas de expresión con variables que son consideradas como causas y consecuencias de los patrones de ubicación de la población en la ciudad. Se realizan pruebas estadísticas que determinan su alta concentración y la correlación entre estas dos variables.

Palabras clave

calidad de vida; segregación residencial; precios del suelo; análisis factorial 


\title{
Quality of life and its Correlation with Land Prices:
}

\author{
An Approach to Residential Segregation in Bogota
}

Abstract This article aims to characterize by means of empirical data the concentration of high and low quality of life conditions in Bogota in 2005, as well as to geo-reference the land prices in a scale of high geographical proximity. The aforementioned will be seen under the light of the concept of residential segregation, in an attempt to identify its forms of expression with variables that are considered as the causes and consequences of the population location patterns in the city. We performed statistical tests that determine its high concentration and the correlation between these two variables.

Keywords quality of life; residential segregation; land prices; factor analysis

\section{Qualidade de vida e sua correlação com os preços do solo: \\ aproximação à segregação residencial em Bogotá}

Resumo Com o artigo visa-se caracterizar, mediante dados empíricos, a concentração de condiçóes de alta e baixa qualidade de vida na cidade de Bogotá em 2005, bem como o georreferenciamento dos preços do solo, em uma escala de larga proximidade geográfica. Isso, à luz do conceito de segregação residencial, visando identificar suas formas de expressão com variáveis que são consideradas causas e consequências dos padróes de localização da populaçáo na cidade. Realizam-se testes estatísticos que determinam a alta concentraçáo e a correlação entre essas duas variáveis. 


\section{Introducción}

La segregación residencial es un tema de interés para distintas disciplinas, en especial en las ciudades latinoamericanas que se insertan en contextos tan desiguales social y económicamente. En el marco de los estudios urbanos, el concepto remite a la distribución de grupos de población en el territorio, que se manifiesta tanto por la proximidad entre los espacios residenciales de los grupos como por la homogeneidad social de las zonas en las que se organiza una ciudad o por la concentración de ciertos grupos en áreas específicas de la misma (Rodríguez y Arriagada, 2004). Estos grupos pueden obedecer a distintas características sociales.

En América Latina, el principal motivo asociado con la segregación residencial es el socioeconómico, a diferencia del contexto estadounidense, en donde el tema racial tiene la mayor relevancia (De Souza Briggs, Darden y Aidala; 1999; Jargowsky, 1997) o del contexto europeo, en el cual la procedencia de los hogares resulta fundamental por el fenómeno migratorio (Home Office, 2001; Bayona, 2007; De Esteban, Díaz y Perelló, 2003). Es evidente, entonces, que las diferencias socioeconómicas, raciales y migratorias son los factores que se erigen como fuente de desigualdad, según las características estructurales de las diversas sociedades.

Aunque la segregación residencial pareciera inherente a la vida urbana, en la actualidad preocupa por las consecuencias que parece atraer cuando su causa es socioeconómica, al actuar como una forma de reproducción de las desigualdades de ese tipo. Esto, porque la segregación residencial afecta a los grupos de bajos ingresos e incide en sus capacidades y oportunidades para el acceso a una calidad urbana digna (Marengo y Elorza, 2014).

Así lo han señalado Kaztman (2001) y Dureau, Dupont, Lelièvre, Lévy y Lulle (2003), que han evidenciado el efecto "aislante" generado sobre los pobres urbanos, quienes solo construyen redes sociales con sus pares pobres y reproducen formas de vida y códigos que dificultan la movilidad social ascendente (Rodríguez y Arriagada, 2004). Lo anterior produce una "guetización" de las zonas pobres de la ciudad, que contribuye a la generación de efectos como la concentración de fenómenos de violencia y delincuencia y a la estigmatización de quienes ocupan dichas zonas. Al respecto, Rasse (2015) identifica que el surgimiento de barrios de pobreza homogénea, sobre todo localizados en las periferias de las ciudades latinoamericanas, tiene como resultado el surgimiento de diversos problemas sociales que conllevan a un deterioro de la calidad de vida de los hogares allí asentados.

La literatura sobre segregación residencial diferencia entre segregación objetiva y subjetiva (Sabatini, 2006). Se concluye que existen unos factores objetivos sobre los cuales se construyen imaginarios de segregación en el espacio, entre los que se identifican dos: las condiciones de vida de la población y las dinámicas del mercado inmobiliario. La relación de estos dos elementos es fundamental para comprender las lógicas de segregación, sus consecuencias y el potencial papel del Estado en este panorama. Sin embargo, cada elemento tiene implicaciones conceptuales de mayor complejidad y merecen ser mencionadas. 
Desde el lado de las condiciones socioeconómicas, han sido recurrentes las referencias a la llamada teoría del espejo, frente a la que parece haber consenso, ya que plantea que el espacio urbano refleja las desigualdades sociales. Esta forma de interpretar el vínculo entre lo social y lo espacial se ha puesto en duda en numerosos estudios comparativos, como el realizado entre las ciudades de Brasil y las de Estados Unidos, que mostró a las primeras más desiguales y a las segundas, más segregadas (Sabatini y Brain, 2008). En este sentido, queda planteada la pregunta por la posibilidad de que cuantas menores diferencias sociales, mayor segregación. Como sea que funcione, lo que este postulado deja claro es que tanto la estructura social como la espacial son cambiantes y tienen una interrelación, que puede contribuir a la conformación de identidades en el espacio, con sus ventajas y desventajas (Sabatini, Cáceres y Cerdá, 2001).

En cuanto a las dinámicas inmobiliarias, en consonancia con el planteamiento de la segregación objetiva y subjetiva, surge la pregunta por los motivos que tienen las personas para elegir su lugar de residencia. En algunos casos, se argumenta que uno de los principales criterios es la posibilidad de vivir cerca de personas similares, es decir, estar rodeado de pares con quienes se logre construir una identidad. Sin embargo, este argumento solo se demostraría en aquellos contextos en los que el mercado inmobiliario tuviera una importancia secundaria y se permitiera el acceso relativamente igualitario al mercado del suelo. En el contexto latinoamericano, la uniformidad social del espacio como una de las características identificables de la situación de segregación de los grupos socioeconómicos bajos dista de ser un producto de la agregación de las decisiones libres de localización (Loaiza y Carvajal, 2014). Más bien, parece vinculada a la dificultad de acceder a suelo barato en donde desarrollar procesos de urbanización con viviendas adecuadas y dotaciones suficientes (Mier, Vásquez y Ziccardi, 2012).

Algunos autores aseguran que en América Latina la ciudad está dividida en dos: una para quienes pueden pagarla, con acceso a bienes y servicios de calidad y con un Gobierno que garantiza la prestación de servicios sociales; y otra para quienes no tienen capacidad de pago, con carencias y déficits en el acceso a bienes y servicios urbanos (Loaiza y Carvajal, 2014).

En Bogotá se han hecho ejercicios de estimación de los índices de segregación, con numerosas limitaciones en el campo de la disponibilidad y escala de los datos, pero con interesantes conclusiones. Por un lado, se encuentran las investigaciones de la Secretaría Distrital de Planeación y la Universidad Nacional de Colombia (2007; 2013), que elaboran clasificaciones socioeconómicas sobre los resultados de la encuesta de calidad de vida, información territorial de dotaciones públicas y la estratificación de la ciudad ${ }^{1}$. En ellas se hacen estimaciones por localidades ${ }^{2} y$ por $\mathrm{UPZ}^{3}$, las cuales presentan en su interior una heterogeneidad social y urbanística bastante amplia, lo que dificulta la lectura de la incidencia territorial del fenómeno de la segregación. Por otro lado, se identifican trabajos que infieren condiciones de calidad de vida y su distribución espacial a partir de los estratos (Aliaga y Álvarez, 2010), otros que calculan índices de condiciones de vida a partir de los años de educación de la población (Salas, 2008) y algunos que ofrecen indicadores de calidad de vida por localidades (Ríos, 2010).

Índice elaborado por la Administración de la ciudad, con el que se evalúa la calidad de las viviendas y el entorno urbano. Asigna un número de estrato del 1 al 6 a un grupo de viviendas que, por lo general, corresponde a una manzana: cuanto más alto sea el número, más alto es el estrato (Aliaga y Álvarez, 2010).

División del territorio de Bogotá en veinte unidades administrativas para la gestión y ejecución de programas y proyectos locales.

3 División del territorio de Bogotá en 114 unidades de planeamiento zonal, adoptadas por el Plan de Ordenamiento Territorial para la formulación de normas urbanísticas de usos del suelo y normas de construcción y proyectos de infraestructura local. 
Ubicado en este estado de la cuestión, a partir de datos estadísticos, el presente artículo busca identificar si las condiciones de mercado inmobiliario están asociadas con la calidad de vida en Bogotá, relación desarrollada en la literatura, pero escasamente soportada de forma empírica.

En la primera sección, se adelanta un ejercicio de medición de la calidad de vida de manera espacializada, lo cual plantea el reto metodológico de producir información a un alto grado de desagregación territorial, de manera que se evidencie la heterogeneidad de las unidades administrativas que tradicionalmente se utilizan como unidad de análisis. En la segunda, se explora la distribución de los precios del suelo en la ciudad y, por último, se muestran las relaciones estadísticas entre las dos variables mencionadas, para establecer el impacto que puede tener el mercado inmobiliario sobre la distribución y la calidad de vida de los grupos sociales. Cada aparte presenta una introducción conceptual seguida de la descripción metodológica y de los resultados hallados.

\section{Calidad de vida espacializada: ¿Se cumple la teoría del espejo?}

Como ya se advirtió, abordar la teoría del espejo en la ciudad implica medir la segregación residencial por factores socioeconómicos, para lo cual se requiere establecer categorías, interés que han compartido desde hace décadas varias de las ramas de las Ciencias Sociales que se han enfocado a la medición de la calidad de vida.

A partir de las diversas definiciones de pobreza o calidad de vida que se asocian con nociones que van desde necesidades o carencias hasta capacidades se han generado diversos métodos dedicados a su medición directa (observación de los bienes físicos y características particulares de los hogares) o indirecta (identificación del ingreso), por mencionar solo una de las clasificaciones existentes (Feres y Mancero, 2001).

No es el objetivo de este artículo profundizar en las ventajas y desventajas de los modelos conceptuales y metodológicos desarrollados hasta el momento, pero sí corresponde advertir sobre un problema que comparte la mayoría de ellos, en función del análisis del comportamiento espacial de la calidad de vida, es decir, del estudio de la segregación residencial. Todos los indicadores de calidad de vida para la ciudad están disponibles en una escala meso que, para este caso, corresponde a las localidades ${ }^{4}$. Lo anterior implica que el escenario micro está desprovisto de indicadores de calidad de vida agregados.

El problema de escala resulta crucial, dado que, según Sabatini (2006), una de las dimensiones de la segregación es el grado de concentración espacial de los grupos sociales o, visto desde otra óptica, la homogeneidad social de las áreas internas de la ciudad. Afirma que la segregación no tiene consecuencias negativas per se, sino que son más intensas cuando existe menor integración entre grupos sociales.

El aislamiento espacial de los pobres o discriminados, y la percepción que ellos tienen de esa condición, es lo que favorece la desintegración social. Por lo tanto, si la segregación ocurre en una escala geográfica reducida, como una ciudad de pequeño tamaño o a través de la conformación de pequeños vecindarios socialmente homogéneos, los efectos negativos de la segregación pueden ser menores o, incluso, no existir. Cuando, en cambio, la segregación se hace intensa en escalas agregadas, rebasándose los márgenes de lo "caminable" y restringiéndose las posibilidades de interacción física entre los grupos sociales, la segregación espacial puede volverse negativa, especialmente para los pobres (p. 9).

División administrativa de la ciudad con base en parámetros de proximidad geográfica. Bogotá se divide en diecinueve localidades urbanas y una rural, que funcionan bajo un esquema de desconcentración administrativa. 


\section{Cálculo del indicador}

Cuantificar la relación entre los dos factores objetivos de la segregación no es una tarea sencilla, pues requiere de una serie de decisiones de orden metodológico vinculadas a tres cuestiones fundamentales: la definición de las categorías o los grupos sociales; la decisión de las unidades espaciales a utilizar y la elección de medidas estadísticas para cuantificar la magnitud del fenómeno (Rodríguez, 2013).

Tiene especial importancia la selección de las unidades espaciales para cuantificar la dimensión de la segregación, porque la investigación social ha mostrado que los indicadores no espaciales tienden a invisibilizar el principal aspecto de la segregación, es decir, el aislamiento de grupos en el espacio. Se trata de un craso error, ya que la segregación es un fenómeno espacial por excelencia (Garroncho y Campos-Alanís, 2013).

Para calcular un indicador que identifique a los grupos sociales por calidad de vida en una escala territorial más próxima, se procedió a espacializar

Tabla 1.

Dimensiones e indicadores/variables a considerar

\begin{tabular}{|c|c|}
\hline Dimensiones & Indicador/variable \\
\hline Hacinamiento no mitigable & Hogares por vivienda \\
\hline Tamaño del hogar & Promedio de personas por hogar \\
\hline \multirow{3}{*}{ Cobertura de servicios públicos } & Tasa de hogares sin servicio de acueducto \\
\hline & Tasa de hogares sin servicio de alcantarillado \\
\hline & Tasa de hogares sin servicio de energía \\
\hline Tipología de vivienda & Tasa de hogares en viviendas tipo cuarto \\
\hline \multirow{2}{*}{ Actividad desarrollada por los integrantes del hogar } & Tasa de dependencia económica \\
\hline & Tasa de población mayor de 17 años sin empleo \\
\hline \multirow{2}{*}{ Grado educativo alcanzado por los integrantes del hogar } & Promedio de años de estudio alcanzado \\
\hline & Tasa de población mayor de 17 años sin educación \\
\hline Sin consumo de alimentos en la última semana & $\begin{array}{l}\text { Tasa de personas que no consumieron ninguna de las tres comidas algún } \\
\text { día de la última semana }\end{array}$ \\
\hline
\end{tabular}

la información disponible del censo del año 2005 por sectores censales. En primer lugar, se consultó el módulo del censo básico del Redatam del Departamento Administrativo Nacional de Estadística (DANE), ya que dispone de información de todas las unidades territoriales (manzana, sección, sector, localidad) y se evaluaron las variables disponibles para construir indicadores de logro que se pudieran sintetizar en un índice de calidad de vida básico.

De acuerdo con dicha disponibilidad y el conocimiento de las variables que tradicionalmente se asocian con la calidad de vida urbana, se seleccionaron siete dimensiones referidas tanto a factores demográficos como de calidad residencial y de capital humano que, aunque no son exhaustivas, han sido abordadas en diversas metodologías de medición de la calidad de vida.

Con estas dimensiones se construyeron los indicadores que se presentan en la Tabla 1, los cuales serán tomados como variables en las pruebas estadísticas. En todos los casos, estos indicadores presentan una escala negativa, ya que cuanto mayor es el indicador, peor es la calidad de vida del sector censal. 
Con base en estas variables, se aplicó un análisis factorial para producir un índice compuesto que contenga el peso de cada una. El análisis factorial es una técnica estadística multivariada, cuyo objetivo es la definición de una estructura subyacente en una matriz de datos. Esta técnica permite estudiar la estructura de las correlaciones entre variables y casos y definir un número de factores comunes y subyacentes. Como lo afirman Navarro, Venegas y Zamora (2014), la técnica permite estudiar la dependencia entre las variables y la asociación entre atributos, para una revisión de la intensidad de las atracciones y repulsiones entre las modalidades que pueden presentar las características cualitativas a partir del estudio de las frecuencias conjuntas observadas.

Previo a la aplicación del análisis factorial, los indicadores fueron estandarizados y homogenizados en una escala de 1 a 100 mediante el siguiente procedimiento (Ecuación 1):
Ecuación 1

Modelo de estandarización de datos

$$
E_{j}=\frac{S_{j} \operatorname{Max}(S)}{\operatorname{Max}(S)-\operatorname{Min}(S)} \times 100 \times(-1)
$$

Fuente: elaboración propia

Además, se tomaron decisiones sobre las variables a incluir a partir de una revisión de las correlaciones bivariadas entre ellas, tras aplicar una prueba de hipótesis con nivel de significancia de $95 \%$. Se decidió excluir la variable porcentaje de viviendas tipo cuarto porque no tenía correlación por lo menos con un $70 \%$ de las variables.

En el modelo se redujeron las diez variables a tres factores, con lo que se logró una explicación de $77 \%$ de la varianza; esto permite afirmar que el modelo es válido 5 .

Tabla 2.

Varianza total explicada

\begin{tabular}{|c|c|c|c|c|c|c|c|c|c|}
\hline \multirow{2}{*}{$\begin{array}{l}\text { 를 } \\
\text { 등 } \\
\text { ․ㅡㄹ } \\
\text { ठ }\end{array}$} & \multicolumn{3}{|c|}{ Autovalores iniciales } & \multicolumn{3}{|c|}{$\begin{array}{l}\text { Sumas de las saturaciones al cuadrado } \\
\text { de la extracción }\end{array}$} & \multicolumn{3}{|c|}{$\begin{array}{l}\text { Suma de las saturaciones al cuadrado } \\
\text { de la rotación }\end{array}$} \\
\hline & Total & $\begin{array}{c}\% \text { de la } \\
\text { varianza }\end{array}$ & $\begin{array}{l}\% \text { acumu- } \\
\text { lado }\end{array}$ & Total & $\begin{array}{c}\% \text { de la } \\
\text { varianza }\end{array}$ & $\begin{array}{l}\% \text { acumu- } \\
\text { lado }\end{array}$ & Total & $\begin{array}{c}\% \text { de la } \\
\text { varianza }\end{array}$ & $\%$ acumulado \\
\hline 1 & 4,86 & 48,603 & 48,603 & 4,86 & 48,603 & 48,603 & 3,147 & 31,474 & 31,474 \\
\hline 2 & 1,639 & 16,39 & 64,993 & 1,639 & 16,39 & 64,993 & 2,396 & 23,956 & 55,43 \\
\hline 3 & 1,201 & 12,009 & 77,001 & 1,201 & 12,009 & 77,001 & 2,157 & 21,571 & 77,001 \\
\hline 4 & 0,683 & 6,831 & 83,832 & & & & & & \\
\hline 5 & 0,45 & 4,504 & 88,336 & & & & & & \\
\hline 6 & 0,405 & 4,053 & 92,389 & & & & & & \\
\hline 7 & 0,25 & 2,503 & 94,892 & & & & & & \\
\hline 8 & 0,213 & 2,134 & 97,026 & & & & & & \\
\hline 9 & 0,172 & 1,718 & 98,744 & & & & & & \\
\hline 10 & 0,126 & 1,256 & 100 & & & & & & \\
\hline
\end{tabular}

Fuente: elaboración propia

Con las variables seleccionadas se obtuvo un KMO de 0,837, el cual puede ser considerado alto y permite una aceptable adecuación muestral. Asimismo, se comparó la prueba de esfericidad de Bartlett, con un $\mathrm{P}$ valor $(0,00)<0,05$ que rechaza la hipótesis nula y acepta la alterna, por lo que el modelo es válido. 
Al aplicar una rotación para mejorar la distribución de las correlaciones entre las variables y los factores, se obtiene que el factor 1 explica un $31,4 \%$ de la varianza total de las variables; el factor 2 , un $23,9 \%$ y el factor 3 , un $21,5 \%$. De tal forma, se puede afirmar que es un modelo equilibrado en el que si bien el peso de los factores muestra una mayor importancia del 1 , no duplica en peso a los otros dos.

Las comunalidades del modelo indican que todas las variables están explicadas en más de un $60 \%$ en los tres factores, como se puede notar en la Tabla 3.

Tabla 3.

Comunalidades

\begin{tabular}{|ccc|}
\hline & Inicial & Extracción \\
\hline Personas por hogar & 1 & 0,694 \\
\hline Hogares por vivienda & 1 & 0,599 \\
\hline Población que ayunó & 1 & 0,803 \\
\hline Promedio de años de educación & 1 & 0,788 \\
\hline Índice de dependencia económica & 1 & 0,856 \\
\hline Porcentaje de población en desempleo & 1 & 0,771 \\
\hline Porcentaje de población sin educación & 1 & 0,869 \\
\hline Porcentaje de viviendas & 1 & 0,735 \\
\hline sin energía eléctrica & & 0,75 \\
\hline Porcentaje de viviendas sin acueducto & 1 & 0,835 \\
\hline Porcentaje de viviendas & 1 & \multirow{2}{*}{ sin alcantarillado } \\
\hline
\end{tabular}

Fuente: elaboración propia

A partir de las correlaciones de cada variable con los factores (Tabla 4) y los resultados del gráfico rotado (Figura 1) de los componentes se obtiene una definición de los factores.

El factor 1 explica un 31,4\% de la varianza de las variables. Sus componentes principales son el ayuno, el promedio de años de educación de la población, el porcentaje de población sin
Tabla 4.

Matriz de componentes rotados ${ }^{6}$

\begin{tabular}{|c|c|c|c|}
\hline & \multicolumn{3}{|c|}{ Componente } \\
\hline & 1 & 2 & 3 \\
\hline Personas por hogar & 0,128 & 0,1 & 0,817 \\
\hline Hogares por vivienda & 0,083 & 0,053 & 0,768 \\
\hline Población que ayunó & 0,852 & 0,221 & 0,166 \\
\hline $\begin{array}{l}\text { Promedio de años } \\
\text { de educación }\end{array}$ & 0,732 & 0,21 & 0,457 \\
\hline $\begin{array}{l}\text { Índice de dependencia } \\
\text { económica }\end{array}$ & 0,607 & 0,218 & 0,663 \\
\hline $\begin{array}{c}\text { Porcentaje de población en } \\
\text { desempleo }\end{array}$ & 0,874 & 0,021 & $-0,083$ \\
\hline $\begin{array}{l}\text { Porcentaje de población } \\
\text { sin educación }\end{array}$ & 0,782 & 0,267 & 0,431 \\
\hline $\begin{array}{l}\text { Porcentaje de viviendas } \\
\text { sin energía eléctrica }\end{array}$ & 0,257 & 0,807 & 0,131 \\
\hline $\begin{array}{l}\text { Porcentaje de viviendas } \\
\text { sin acueducto }\end{array}$ & 0,003 & 0,862 & 0,085 \\
\hline $\begin{array}{l}\text { Porcentaje de viviendas } \\
\text { sin alcantarillado }\end{array}$ & 0,228 & 0,881 & 0,085 \\
\hline
\end{tabular}

Fuente: elaboración propia

educación y el porcentaje de población desempleada. Dado que se compone de características de los individuos y su inserción en la estructura económica, se denomina Factor socioeconómico.

El factor 2 explica un 23,9\% de la varianza de las variables. Sus componentes principales son el porcentaje de viviendas con energía eléctrica, el porcentaje de viviendas con servicio de acueducto y el porcentaje de viviendas con servicio de alcantarillado. Puesto que se conforma por características de las viviendas por la presencia de servicios públicos, se denomina Factor de servicios públicos en la vivienda.

El factor 3 explica un 21,5\% de la varianza de las variables. Sus componentes principales son el número de hogares por vivienda, el número de personas por hogar y el indicador de dependencia

6 La matriz de componentes rotados, en este caso por el método Varimax, presenta las correlaciones de cada variable con los factores, de manera tal que permite identificar la asociación del grupo de variables que definen cada factor. La identificación de las correlaciones más altas en cada factor conduce a la denominación del mismo. 
económica. Ya que se compone de características de la población con respecto a su estructura etaria, la composición del hogar y la distribución de viviendas en los hogares, se denomina Factor de composición demográfica.

Figura 1.

Gráfico rotado de los componentes

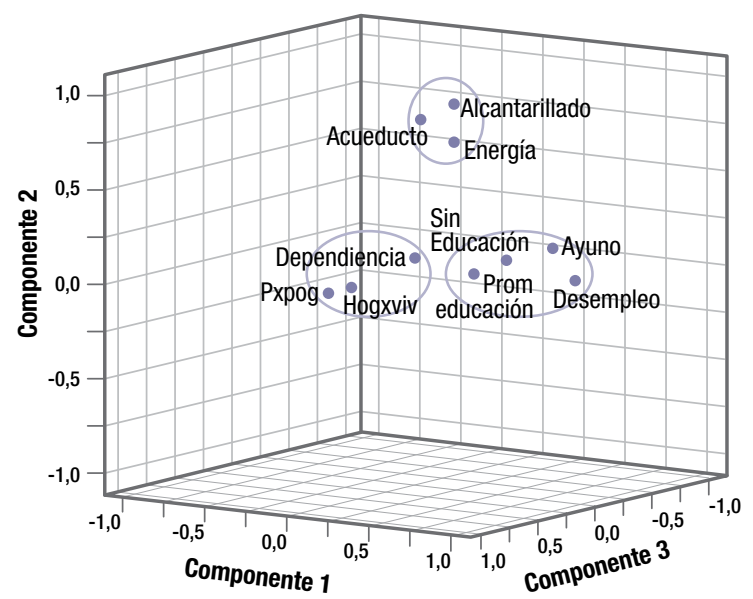

Fuente: elaboración propia

Por último, para obtener un índice con base en los tres factores, dado que en ellos ya están los pesos de las variables ${ }^{7}$, el índice se concibe como un promedio en el que cada factor tiene el mismo peso (Ecuación 2).

Ecuación 2.

Modelo de ponderación de factores (ICV básico)

$$
I C V=f_{1}+f_{2}+f_{3}
$$

En donde:

$$
\begin{aligned}
& f_{1}=\text { características socioeconómicas } \\
& f_{2}=\text { servicios públicos en la vivienda } \\
& f_{3}=\text { composición demográfica }
\end{aligned}
$$

Fuente: elaboración propia

Al espacializar los resultados del ICV básico por sectores censales en Bogotá, se obtiene una distribución que presenta un patrón fuerte de concentración de condiciones favorables hacia el área central de la ciudad y de dispersión de condiciones adversas en distintas intensidades hacia la periferia (Figura 2).

Se observa que las condiciones más bajas de calidad de vida predominan en la ciudad, aproximadamente en un $50 \%$ de los sectores censales (262 de los 564 sectores evaluados) con el menor rango del índice. Esta tendencia se presenta con mayor intensidad hacia la zona sur de la ciudad, en las localidades de San Cristóbal, Usme, Rafael Uribe, Tunjuelito, Ciudad Bolívar y Bosa, en donde las bajas condiciones predominan en un $90 \%$ de los sectores que los componen. En estas áreas, se observa la homogeneidad socioeconómica que abarca varias localidades y que supera la escala de "lo caminable", lo que evidencia la consolidación de un polo de segregación marcada en la ciudad.

Los valores intermedios se encuentran en la zona central y hacia los bordes noroccidentales de la ciudad, en 196 sectores censales que representan un $34 \%$ de los sectores evaluados y se distribuyen en las localidades de Antonio Nariño, Mártires, Puente Aranda, Kennedy, Fontibón, Engativá, Barrios Unidos y, en menor medida, Suba. Resulta de particular interés el hecho de que en estos sectores se entremezclan valores medios y bajos, con una tendencia predominante de valores medios, que señalan una variación progresiva de la tendencia general de aumento de calidad de vida hacia la zona central.

Existe una centralidad consolidada de condiciones altas hacia el centro y nororiente en 106 sectores censales que representan solo un $18 \%$ de los sectores evaluados y se encuentran hacia Chapinero, Teusaquillo y Usaquén, con una

Los pesos de las variables se obtienen de las puntuaciones factoriales que estiman el valor de cada factor para cada sector censal mediante el método de regresión. Se producen valores con media de 0 y una varianza igual al cuadrado de la correlación múltiple entre las puntuaciones factoriales estimadas y los valores factoriales verdaderos. 
Figura 2.

Espacialización del ICV básico por sectores censales. Bogotá, 2005

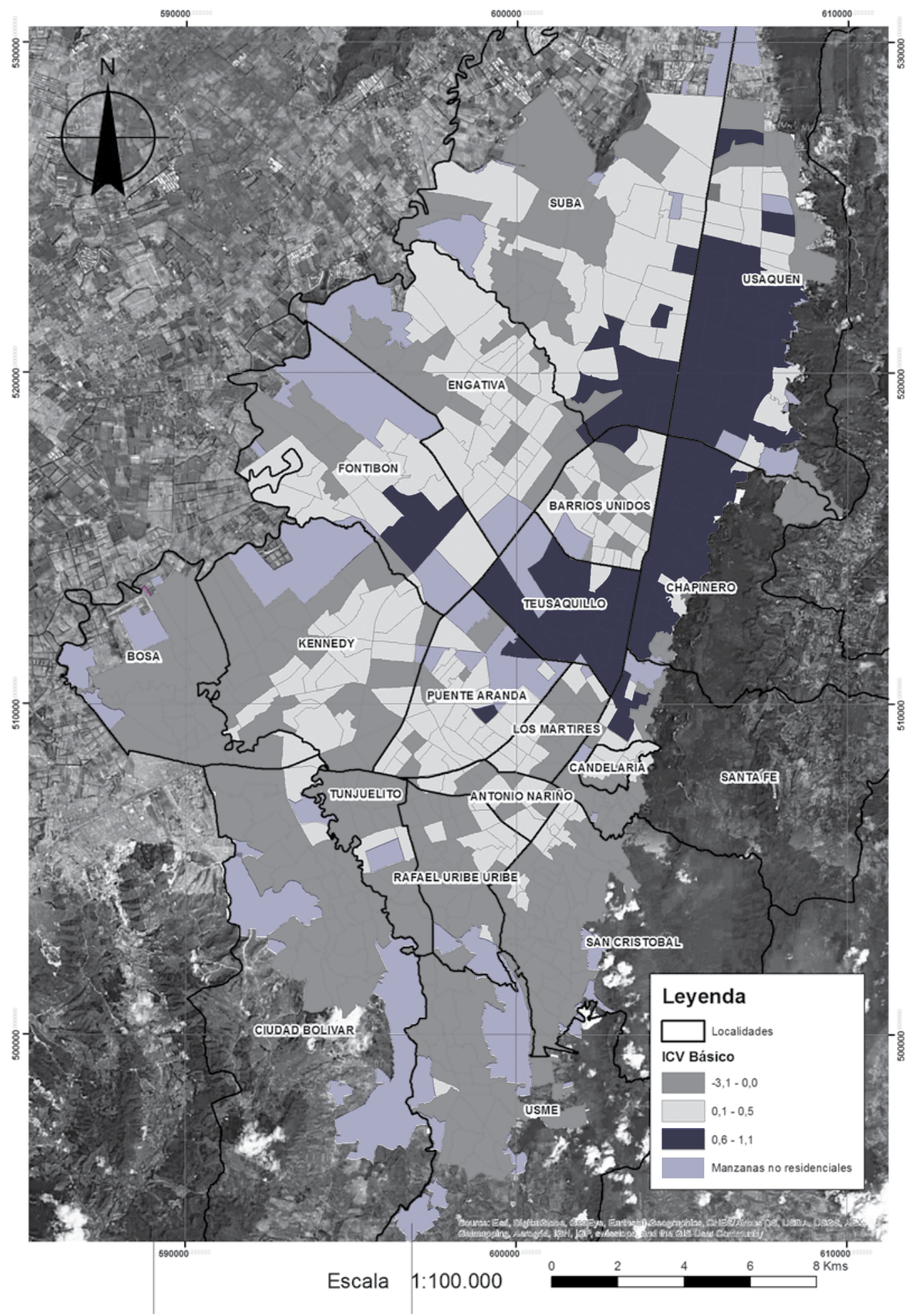


tendencia predominante de condiciones altas agrupadas hacia los cerros orientales. Asimismo, se destacan algunos sectores censales de Santa fe, Fontibón, Suba y Puente Aranda que, por su proximidad con estas zonas, presentan dinámicas similares en los sectores cercanos a la centralidad.

Ofrecen especial interés dos fenómenos que tienen lugar en este patrón: en primer lugar, se identifican algunas zonas en donde la variabilidad de valores obtenidos denota alta heterogeneidad de condiciones de vida que coexisten en zonas de alta proximidad; se ubican en esta categoría los sectores de Usaquén, Suba y la zona centro, en particular hacia Santa fe. En segundo lugar, es importante destacar el comportamiento que tienen el eje vial de la avenida Caracas y la autopista Norte, entre las calles 26 y 170 , que funciona como eje articulador de las zonas con mejores condiciones de vida en la que, como un comportamiento atípico, se registra el de la localidad de Barrios Unidos (al costado occidental del eje entre las calles 63 y 100), próxima a las concentración de valores óptimos, pero con predominio de los valores medios y bajos.

Para evaluar la tendencia espacial del ICV, se aplicó un prueba de hipótesis de la autocorrelación espacial global denominada índice de Moran ${ }^{8}$. Con un valor de correlación espacial de 0,46 y un valor $P$ inferior a 0,05 , se acepta que el comportamiento tipo clúster del ICV básico es estadísticamente significativo. Por ello, se considera que la distribución espacial de los valores altos y los valores bajos en el conjunto de datos está más agrupada espacialmente de lo que se esperaría si los procesos espaciales fueran aleatorios.

Se concluye, entonces, que el fenómeno de la pobreza en Bogotá sigue un patrón espacial determinado que presenta distintos índices de segregación según la zona. Se consolida un amplio eje sur, altamente homogéneo, en el que se inserta cerca de un $50 \%$ de los sectores analizados y parece conformar un patrón de segregación de escala intermedia. Sin embargo, la heterogeneidad también tiene su lugar en sectores ampliamente disímiles como los que existen en la zona norte de la ciudad, en donde la segregación se estaría presentando en una escala micro. Allí sería del mayor interés identificar los mecanismos de diferenciación y convivencia de los grupos sociales diferenciados.

\section{La localización en la ciudad: ¿Cuestión de acceso al mercado inmobiliario 0 autoconstrucción de identidades?}

Para Sabatini y Brain (2008), en América Latina el funcionamiento de los mercados del suelo produce niveles de segregación que exceden los que podrían justificarse por la preferencia de las personas, lo que lleva a entender la segregación espacial por motivos socioeconómicos más como una expresión de la "capitalización de plusvalías" que como un mecanismo de defensa de las identidades ligadas a las diferencias socioeconómicas. Esto quiere decir que en la conformación y

8 La noción de autocorrelación espacial se vincula con la idea de que valores observados en áreas cercanas o adyacentes serán más similares que los esperados bajo el supuesto de independencia espacial o de aquellos más lejanos (Paradis, 2015). La fórmula del coeficiente I de Moran es:

$$
I=\frac{n}{S_{0}} \frac{\sum_{j}^{n}=1 w_{i, j} z_{i} z_{j}}{\sum_{i}^{n}=1 z_{i}^{2}}
$$

En donde $n$ significa el número de las unidades (áreas o puntos) en el mapa $W_{i j}$ es la matriz de distancias que define si las áreas o puntos geográficos, iy j, son vecinos o no. $Z_{i}$ es la desviación de un atributo del elemento $i$ de su media $\left(X_{i}-\bar{X}\right)$ y $S_{0}$ es el agregado de todas distancias espaciales (Vilalta y Perdomo, 2005). 
continuidad de la segregación en nuestros contextos parecen primar los factores objetivos $y$ estructurales de urbanización de la ciudad por encima de los factores subjetivos que conducen al autoaislamiento.

Esta condición empezó a tomar forma en las principales ciudades latinoamericanas a partir de su inserción en el sistema competitivo de economía especializada de escala global, que tuvo lugar sobre todo en la década del ochenta, lo cual las ha expuesto a grandes inversiones estructurales y sociales que no han resultado del todo equilibradas, pues las principales metrópolis de la región están lejos de resolver sus contradicciones (Brand, 2009). En este contexto, fenómenos como la fuerte acumulación de riquezas y los altos flujos migratorios, sumados al deseo de mantener un crecimiento económico constante e ilimitado y a las políticas de desregularización de los mercados de suelo, han profundizado las condiciones de segregación residencial.

Desde esta lógica económica y política que ha determinado la configuración de los mercados de suelo e inmobiliarios, la vivienda ha constituido un objeto de consumo que adquiere un valor de uso - dado por su potencial de albergue- y un valor de cambio - que se determina tanto por su costo de producción como por su localización respecto a las demás estructuras de la ciudad-.

Así lo señala David Harvey (2015), al plantear que el costo de adquisición de la vivienda está determinado por las materias primas y el trabajo, el margen de beneficio que espera el productor y el costo del suelo; este último es el que se ve afectado por los fenómenos de diferenciación y segregación urbana. En el mismo sentido, Samuel Jaramillo (2009) propone una renta del suelo determinada por dos elementos: las rentas primarias, que surgen alrededor del proceso productivo de la construcción y las rentas secundarias, que se derivan del espacio urbano construido en su proceso de su consumo, en el que resulta determinante la localización.

Aparece una aproximación a la renta diferencial del suelo asociado con las condiciones de proximidad a infraestructuras urbanas y centros de producción económicos. Así lo señala el mismo autor:

La vivienda es un bien que para poder ser consumido cabalmente debe estar enlazado espacialmente con otros valores de uso complementarios: los sitios de aprovisionamiento, los lugares de reproducción colectiva de la fuerza de trabajo y, por supuesto, los lugares de empleo. Ahora bien, la heterogeneidad característica del espacio urbano determina que no todos los lugares destinados a la vivienda obrera estén entrelazados de manera idéntica con estos valores de uso complementarios [...]. De esta manera, surge el hecho de que las distintas ubicaciones significan costos diferentes para apropiarse de valores de uso comparables (Jaramillo, 2009, p. 167).

Producto de la misma naturaleza compleja del espacio, el comportamiento del mercado de suelo y, por ende, el mismo valor de la vivienda fluctúan según múltiples factores; la especulación es uno de ellos, así como lo son los valores de cambio de las unidades cercanas y las características de los grupos sociales que los habitan y sus vecinos. Es por ello que clases más acomodadas evitan la cercanía de los más pobres y argumentan que esto dificultaría la valorización de sus inmuebles.

En suma, en ciudades como Bogotá, la provisión de vivienda ha desplazado el interés en la producción de valores de uso por la búsqueda del aumento del valor de cambio y la convierte en un objeto de especulación, en mercancía para vender en un mercado al costo de quien pueda pagarla, lo que explica por qué existen ofertas de vivienda para diferentes grupos socioeconómicos (Harvey, 2015). De allí se deriva una relación ingreso-localización de residencia en la que los bajos ingresos afectan las posibilidades de elección 
de la ubicación y restringen las relaciones espaciales con la ciudad (Brain, Prieto y Sabatini, 2010).

\section{Cálculo del indicador}

Con el objeto de identificar cuán libres podrían ser las elecciones de localización de la vivienda dependiente de los ingresos socioeconómicos, se ha calculado y georreferenciado el promedio del valor del suelo residencial en cada sector censal. Para ello se utilizó como fuente la información catastral de Bogotá del año 2006, de manera que se tuviera cercanía temporal con los datos censales y no se requiriera transpolar los resultados.

El procedimiento utilizado consistió, en primer lugar, en filtrar los predios con uso residencial. Luego se convirtieron a punto (centroide) para hacer un cálculo del promedio del valor del suelo por sector censal mediante una unión espacial y se obtuvieron los resultados presentados en el siguiente plano (Figura 3).

Como resultado, se estima que solo un $10 \%$ de los sectores censales considerados presentan valores promedio superiores a 1.000 .000 de pesos por metro cuadrado, situación que permite identificar el alto grado de desigualdad de distribución de la renta del suelo de la ciudad. Estas zonas se localizan principalmente hacia Chapinero y Usaquén, en las que se destaca el eje de la avenida Caracas y la autopista Norte, ahora entre calles 26 y 151 , a partir del cual se localiza la mayoría de sectores con valores de suelo para uso residencial superiores en promedio a $1.000 .000 \mathrm{de}$ pesos por metro cuadrado. De manera excepcional, se encuentran tres sectores con un comportamiento similar, uno hacia la zona de Fontibón y Teusaquillo, otro en Suba y otro en un área reducida del centro de la ciudad.
Con valores medios comprendidos entre 500.000 pesos y 1.000 .000 de pesos, se encuentra un $40 \%$ de los sectores censales (que corresponde a $226 \mathrm{de}$ los 564 evaluados), ubicados en la zona central y hacia el borde norte y occidental. La distribución de los valores medios en este caso tiene un patrón similar al expuesto en el análisis anterior, pero con una extensión mayor. La tendencia predomina en las localidades de Antonio Nariño, Mártires, Puente Aranda, Rafael Uribe, Fontibón, Engativá, Barrios Unidos y Suba y con menores intensidades en Usaquén y Santa fe.

Por último, con los valores promedio inferiores a 500.000 pesos por metro cuadrado se encuentra un $50 \%$ de los sectores, en 283 de los 564 sectores evaluados. Este comportamiento se concentra hacia la zona sur de la ciudad, en las localidades de San Cristóbal, Usme, Tunjuelito, Ciudad Bolívar y Bosa y agrupaciones en el límite occidental, en Fontibón y Suba. Este último comportamiento se contrapone en términos generales a la noción de una centralidad de valores altos que disminuye su tendencia hacia las periferias, en la medida en que se encuentran dispersos en la mayor extensión de la ciudad sectores censales de bajos valores. Esto permite inferir los procesos de segmentación que han tenido lugar en la ciudad y que inciden en la generación de valores diferenciales de suelo en zonas próximas entre sí.

Mientras tanto, al aplicar la prueba de hipótesis para el índice de Moran, con un valor de correlación espacial de 0,69 y un valor $P$ inferior a 0,05 , se acepta que el comportamiento tipo clúster es estadísticamente significativo, es decir, la distribución espacial de los valores altos y los valores bajos en el conjunto de datos está más agrupada espacialmente de lo que se esperaría si los procesos espaciales fueran aleatorios. La distribución de valores de suelo con destinación residencial en Bogotá para el año 2006 tenía un patrón claro de concentración de valores 
Figura 3.

Distribución de los valores del suelo residencial. Bogotá, 2006

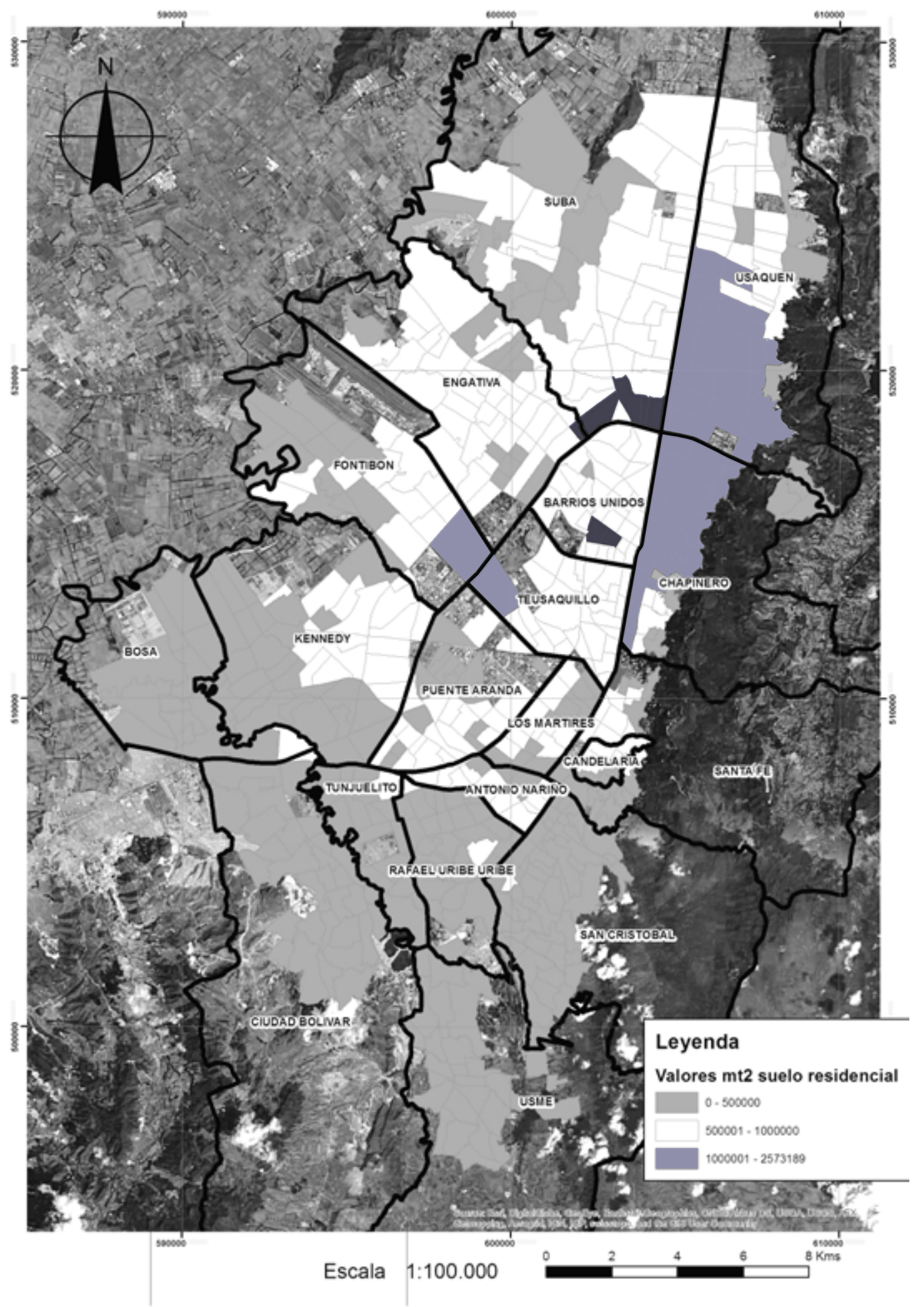


altos hacia la zona oriental, con una tendencia de disminución hacia los bordes perimetrales, que se expresa en comportamientos diferenciales hacia el límite occidental y la zona sur de la ciudad (Figura 3).

Lo anterior permitiría confirmar que el peso del mercado residencial en Bogotá disminuye la posibilidad de escoger libremente la localización de la vivienda y se torna un elemento fundamental para la agrupación en el espacio de personas de las mismas características socioeconómicas.

\section{¿Causas o consecuencias? \\ Relación entre calidad de vida y precios del suelo}

Como ya se observó, tanto las condiciones de calidad de vida en la ciudad como los precios del suelo, presentan patrones geográficos que muestran una alta concentración de grupos sociales similares en la ciudad, lo cual puede interpretarse como un indicador de segregación residencial.

La relación entre estas dos dimensiones de la segregación permite abordar el debate sobre las causas y consecuencias del fenómeno, que parecen cíclicas e interdependientes. Según Sabatini y Brain, (2010), una de las motivaciones de la segregación es la búsqueda de calidad de vida, pues los grupos que pueden elegir la localización de su vivienda buscan el acceso a bienes y servicios a los que suele accederse de manera colectiva: "En medida importante, la conformación de una suerte de cono geográfico de concentración de los grupos altos y medios en la ciudad latinoamericana del siglo XX se puede explicar por estas externalidades de vecindad" (p. 20). De modo simultáneo, la consolidación de áreas aventajadas en términos de calidad de vida refuerza el mecanismo de segregación que se asocia con el mercado inmobiliario.

De allí que se considere como clave la premisa de que "los mercados urbanos producen niveles de segregación residencial más altos que los que resultarían de las preferencias de las personas" (Sabatini, y Brain, 2010, p. 21). Es decir, se producen condiciones de aislamiento superiores a las que los grupos similares generarían para consolidar sus identidades "de clase". Lo anterior afecta en particular a aquellos sin capacidad de pago que, por ende, pierden capacidad de decisión frente a su localización en la ciudad y quedan relegados a tomar las peores opciones en términos de calidad residencial, material y urbana, en muchos casos relacionados con los mercados informales de la vivienda.

Para Bogotá, el estudio de Fuentes (2010) ha seńalado que la demanda efectiva de suelo no ha dependido directamente del crecimiento de la población, sino de las posibilidades económicas de la misma. Señala además que la dinámica de segregación de la ciudad profundiza la precariedad de las condiciones sociales de los más pobres, situación que "es aprovechada por el mercado o los precursores de él, los constructores de vivienda privada, para obtener ganancia a través de proyectos que promueven la segregación socio espacial, vía exclusividad en el uso y acceso a los suelos" (p. 124).

Debido al complejo entramado que se conforma entre calidad de vida y precios del suelo, es relevante identificar las relaciones estadísticas que existen entre estas dos variables para la ciudad de Bogotá, con el fin de establecer empíricamente las condiciones de la segregación residencial actual. 


\section{Cálculo de la correlación}

$\mathrm{Al}$ establecer la correlación entre el índice de calidad de vida básico y los valores promedio del suelo residencial en los sectores censales, con un p valor de 0,000 se acepta que el ICV básico y los valores del suelo residencial sí están correlacionados. El coeficiente de correlación Pearson indica que las dos variables tienen una correlación de 0,657 , la cual puede ser considerada como adecuada para explorar un modelo de regresión.

El modelo de regresión entre las variables indica que hay un mejor ajuste con una curva de tipo logarítmica, dado que el coeficiente de determinación es de 0,588 , mientras en un modelo lineal es de 0,432 . La tendencia logarítmica se puede apreciar en la Figura 4. Se concluye entonces que el ICV básico se puede explicar en un 58,8\% por la distribución de los valores del suelo residencial en la ciudad?.

Puesto que el valor-P en la tabla Anova es menor que 0,05 , existe una relación estadísticamente significativa entre ICV básico y valor metro suelo con un grado de confianza de 95,0\%. De lo anterior se desprende que en Bogotá el ICV básico por sectores censales se puede predecir en función de los precios del suelo, con un modelo logarítmico que tiene la siguiente ecuación:

Ecuación 3.

Ecuación de regresión

$I C V=-8,3628+0,641 * 1 \mathrm{n}$ (valor $\mathrm{m}$ suelo)

Fuente: elaboración propia

\section{Figura 4.}

Gráfico de dispersión entre ICV básico y el promedio del valor del suelo residencial

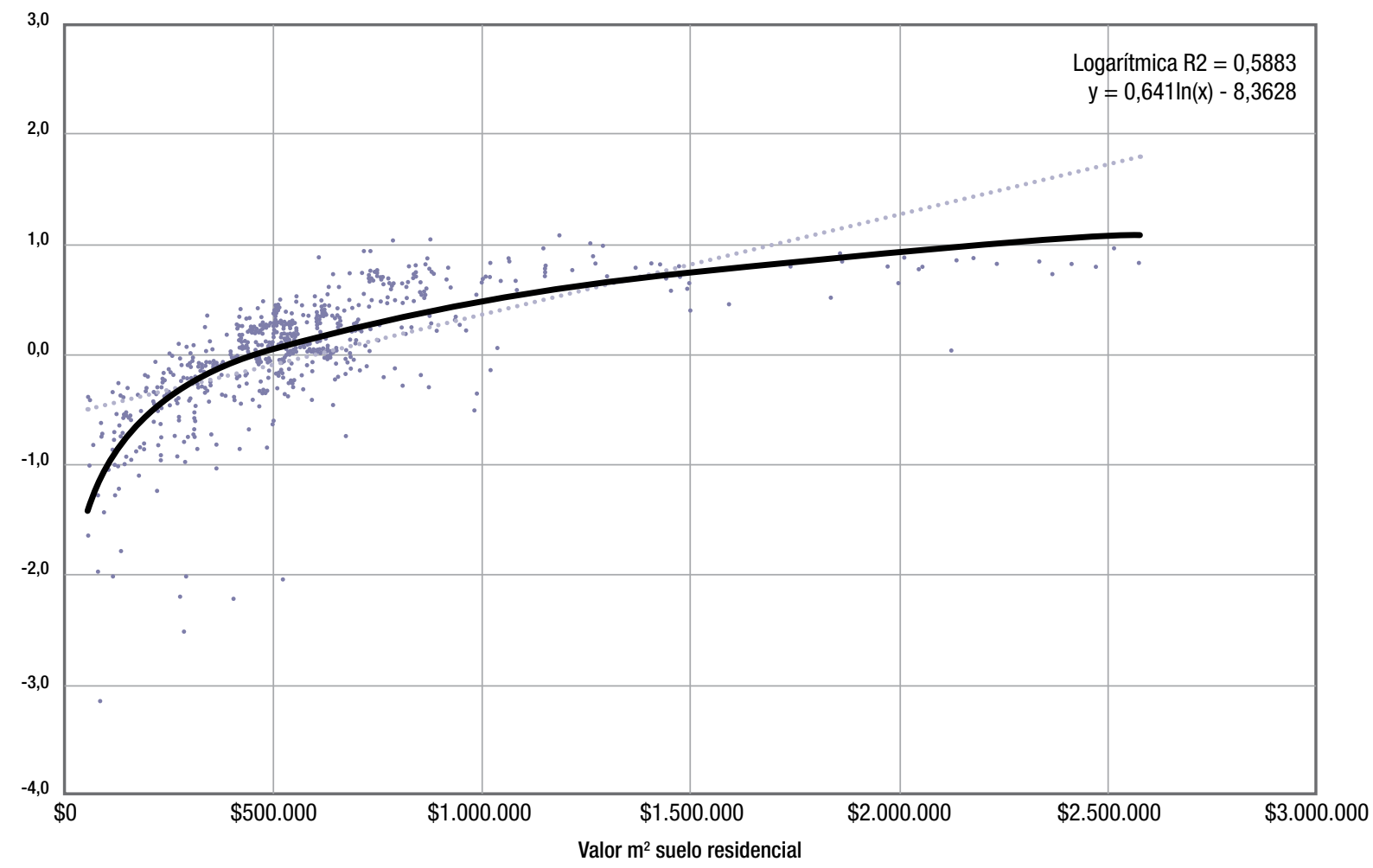

Fuente: elaboración propia

9 Las pruebas de normalidad Kolmogorov-Smirnov y Shapiro-Wilk, ambas con significancia de 0,000, indican que se cumple el supuesto sobre los residuales y tienden a una distribución normal. Con un Durbin Watson de 1,342, las pruebas de independencia de los errores indican que son independientes. De tal forma, se validan los supuestos de normalidad e independencia de los residuales, lo cual señala que el modelo es adecuado. 


\section{Conclusiones}

Con este trabajo se ha generado evidencia empírica sobre tres fenómenos ampliamente estudiados y debatidos en la caracterización de la segregación social y residencial en Bogotá.

El primero de ellos corresponde al análisis del comportamiento espacial que presenta la calidad de vida en la ciudad. Esta caracterización se llevó a cabo en el sector censal, para conseguir detalles mucho más específicos en comparación con la escala meso que representa la localidad, generalmente utilizada para este tipo de revisión. Gracias a esto, se consiguió una mejor aproximación al comportamiento de esta variable y se identificaron los grados de concentración de grupos en condiciones socioeconómicas homogéneas en su interior y un patrón de distribución general en la ciudad más heterogéneo, que tiende a evitar la mezcla de grupos entre sí.

Se encontró que el comportamiento de las condiciones de calidad de vida está altamente sectorizado en la ciudad, en una escala macro generada por la yuxtaposición de sectores micro que, en su conjunto, configuran vastas zonas de la ciudad habitadas por grupos socioeconómicos similares en sus condiciones de vida. Con ello se comprueba que la sociedad bogotana refleja en el espacio construido la magnitud de su segregación y cumple a cabalidad con la discutida teoría del espejo.

Esta teoría también tiene una dimensión temporal que da cuenta de la dinámica de las estructuras sociales y espaciales que no se comprobó en este estudio, porque se trabajó con datos de un solo momento. La comparación con otros escenarios temporales es una línea a desarrollar, para explorar con más riqueza la teoría mencionada.
En segundo lugar, frente al análisis del comportamiento del mercado inmobiliario, se identificó un patrón espacial fuertemente aglomerado que presenta importantes similitudes con la primera variable caracterizada. Con esto se demostró que la segregación tiene una fuerte relación con el comportamiento del mercado inmobiliario, dado que los precios del suelo en la ciudad también están altamente sectorizados.

Este comportamiento refleja una reducción dramática de los grados de libertad con la que cuentan los grupos con menor poder adquisitivo para decidir sobre la localización de sus viviendas y muestra el escaso peso que tiene la voluntad de residir cerca de personas similares, con el fin construir una "identidad de clase"; esto refleja que la segregación en la ciudad se relaciona con las condiciones estructurales más que con deseos de asociación en el territorio.

En tercer lugar, pudo observarse que existe una correlación directa entre la calidad de vida y el valor de suelo en la ciudad, de la cual se obtiene que cuanto mayor sea el valor del suelo, mayor será la calidad de vida. Aunque de entrada esta afirmación parece obvia, en el contexto de las ciudades latinoamericanas, hasta ahora ha sido poco explorada empíricamente, lo que afecta la comprensión de las causas y consecuencias de una segregación residencial con las características ya expresadas. De una parte, este vínculo lleva a reflexionar acerca de las condiciones de vida a las que se ven sometidos los expulsados del sistema inmobiliario $y$, de otra, pone de manifiesto la consolidación de una trampa de pobreza ligada a la "endogamia de clase", que evita el intercambio de capitales entre personas de diferentes grupos socioeconómicos.

En este sentido, es fundamental plantear que las políticas de reducción de la pobreza para la 
ciudad deben tener un componente territorial e ir aparejadas de estrategias de regulación del mercado del suelo vía ordenamiento territorial, cuya premisa sea que en el funcionamiento de los mercados del suelo está la clave para comprender la segregación residencial.

Por último, en términos metodológicos, es importante resaltar el potencial de la aplicación de técnicas estadísticas y de análisis espacial como complemento para el estudio de la segregación residencial, pues en la medida en que se desarrollen análisis en diversas escalas, será posible obtener una aproximación más precisa de su comportamiento, hecho que ha sido uno de los retos más representativos en el estudio del fenómeno. $\mathrm{Al}$ respecto, lograr mediciones en unidades más ajustadas a la heterogeneidad de un territorio permite la estimación de modelos que indiquen la relación compleja entre variables espaciales y variables socioeconómicas, lo cual, a su vez, constituye una herramienta fundamental para el diseño de políticas públicas urbanas que busquen mejorar la calidad de vida de la población.

\section{Bibliografía}

Aliaga, L. y Álvarez, M. (2010). Segregación residencial en Bogotá a través del tiempo y diferentes escalas. Documento de trabajo, Lincoln Institute of Land Policy, Bogotá.

Bayona, J. (2007). La segregación residencial de la población extranjera en Barcelona: ¿Una segregación fragmentada? Scripta Nova, XI(235). Recuperado de http://www.ub.edu/geocrit/sn/ sn-235.htm

Brain, I., Prieto, J. y Sabatini, F. (2010). Vivir en campamentos: ¿Camino hacia la vivienda formal o estrategia de localización para enfrentar la vulnerabilidad? EURE, 36(109), 111-141.

Brand, P. (comp.). (2009). La ciudad latinoamericana en el siglo XXI: globalización, neoliberalismo, planeación. Medellín: Universidad Nacional de Colombia.

De Esteban, A., Díaz, J. y Perelló, S. (2003). Inmigración y segregación urbana. Papeles de economia española (98), 262-273.

De Souza Briggs, X., Darden, J. y Aidala, A. (1999). In the Wake of Desegregation: Early Impacts of Scattered-Site Housing on Neighborhoods in Yonkers, New York. Journal of the American Planning Association, 65(1), 27-49.

Dureau, F., Dupont, V., Lelièvre, É., Lévy, J., y Lulle, T. (2003). Metrópolis en movimiento: una comparación internacional. Bogotá: Alfaomega.

Feres, C. y Mancero, X. (2001). Enfoques para la medición de la pobreza. Breve revisión de literatura. En Cepal (editor de la serie), Estudios estadisticos y prospectivos: vol. 4. Santiago: ONU.

Fuentes, H. (2010). Desigualdad, segregación socio-espacial y precios del suelo en la ciudad latinoamericana. El caso de Bogotá en los años noventa. UIS Humanidades, 38(1), 109-126.

Garroncho, C. y Campos-Alanís, J. (2013). Réquiem por los indicadores no espaciales de segregación residencial. Papeles de Población, 19(77), 269-300.

Harvey, D. (2015). Diecisiete contradicciones y el fin del capitalismo. Quito: IAEN.

Home Office. (2001). A Report of the Independent Review Team Chaired by Ted Cantle. Recuperado de http://dera.ioe.ac.uk/14146/1/communitycohesionreport.pdf 
Jaramillo, G. S. (2009). Hacia una teoría de la renta del suelo urbano. Bogotá: Universidad de los Andes.

Jargowsky, P. (1997). Poverty and Place: Guettos, Barrios, and the American City. Nueva York: Ruseel Sage Foundation.

Kaztman, R. (2001). Seducidos y abandonados: el aisalmento social de los pobres urbanos. Revista de la Cepal, 75, 171-189.

Loaiza, W. y Carvajal, Y. (2014). Índice de segregación espacial y socioeconómico (ISES) en las comunas de Santiago de Cali. Cuadernos de Vivienda y Urbanismo, 7(13), 84-101.

Marengo, C. y Elorza, A. (2014). Tendencias de segregación residencial socioeconómica: el caso de Córdoba (Argentina) en el período 20012008. EURE, 40(120), 111-113.

Mier, A., Vásquez, I. y Ziccardi, A. (2012). Pobreza urbana, segregación residencial y mejoramiento del espacio público en la ciudad de México. Sociologias, 14(30), 118-155.

Navarro, J., Venegas, F. y Zamora, A. (2014). Migración y desarrollo económico en México: un análisis factorial de correspondencias. Migración $y$ desarrollo, 12(22), 123-145.

Paradis, E. (2015). Moran's Autocorrelation Coefficient in Comparative Method. Recuperado de https://cran.r-project.org/web/packages/ape/ vignettes/MoranI.pdf

Rasse, A. (2015). Juntos pero no revueltos. Procesos de integración social en fronteras residenciales entre hogares de distinto nivel socioeconómico. EURE, 41(122), 125-143.

Ríos, M. (2010). Segregación residencial: el problema social desde la perspectiva económica y urbana. (Tesis de Maestría inédita). Universidad Nacional de Colombia, Bogotá.

Rodríguez, G. (2013). El uso de zonas censales para medir la segregación residencial. Contradicciones, propuesta metodológica y un estudio de caso: Argentina 1991-2001. EURE, 39(118), 97-122.

Rodríguez, J. y Arriagada, C. (2004). Segregación residencial en la ciudad latinoamericana. EURE, 30(89), 5-24.

Sabatini, F. (2006). La segregación social del espacio en las ciudades de América Latina. Washington: Banco Interamericano de Desarrollo.

Sabatini, F. y Brain, I. (2008). La segregación, los guetos y la integración social urbana: mitos y claves. EURE, 34(103), 5-26.

Sabatini, F., Cáceres, G. y Cerda, J. (2001). Segregación residencial en las principales ciudades chilenas: tendencias de las tres últimas décadas y posibles cursos de acción. EURE, 27(82), 21-42.

Salas, A. (2008). Residential Segregation and Housing Production in Bogota, between Perceptions and Realities. (Tesis doctoral inédita). Universidad de Poitiers, Francia.

Secretaría Distrital de Planeación y Universidad Nacional de Colombia. (2007). Segregación Socioeconómica en el espacio urbano de Bogotá. Bogotá: Autor.

Secretaría Distrital de Planeación y Universidad Nacional de Colombia. (2013). Segregación Socioeconómica en el espacio urbano de Bogotá. Bogotá: Autor.

Vilalta y Perdomo, C. (2005). Cómo enseñar autocorrelación espacial. Economía, Sociedad y Territorio, 5(18), 323-333. 